\title{
Triticum aestivum sprout-derived polysaccharide exerts hepatoprotective effects against ethanol-induced liver damage by enhancing the antioxidant system in mice
}

\author{
SARMILA NEPALI ${ }^{1}$, HYEON-HUI KI ${ }^{1}$, JI-HYUN LEE ${ }^{1}$, JI-YUN CHA ${ }^{2}$, YOUNG-MI LEE ${ }^{2}$ and DAE-KI KIM ${ }^{1}$ \\ ${ }^{1}$ Department of Immunology and Institute of Medical Sciences, Medical School, Chonbuk National University, \\ Jeonju, Jeonbuk 54907; ${ }^{2}$ Department of Oriental Pharmacy, College of Pharmacy and Wonkwang-Oriental \\ Medicine Research Institute, Wonkwang University, Iksan, Jeonbuk 54538, Republic of Korea
}

Received February 14, 2017; Accepted July 28, 2017

DOI: $10.3892 / \mathrm{ijmm} .2017 .3095$

\begin{abstract}
Triticum aestivum sprout-derived polysaccharide (TASP) has anti-diabetic properties, but no information is available in regards to its protective effect against ethanol-induced hepatic injury. This study aimed to investigate the mechanism behind the protective role of TASP against ethanol-induced liver injury in vivo. Male C57BL/6 mice were administered ethanol with or without TASP for 10 consecutive days by oral gavage. Silymarin was administered in the same manner as a positive control. TASP reduced ethanol-induced hepatic lipid accumulation and serum alanine aminotransferase (ALT) and aspartate aminotransferase (AST) levels. TASP also prevented glutathione (GSH) depletion and increased the superoxide dismutase (SOD) in liver tissue. In addition, TASP significantly inhibited ethanol-induced cytochrome P450 2E1 (CYP2E1) activation, and upregulated the expressions of nuclear factor erythroid 2-related factor 2 (Nrf2) and hemeoxygenase-1 (HO-1), and downregulated NADPH oxidase genes in ethanol fed mice. Furthermore, the upregulation of Nrf2 was found to be regulated by a phosphatidylinositol 3-kinase (PI3K)/Akt pathway. TASP also attenuated hepatic injury by modulation of caspase-3 and apoptosis-associated mitochondrial proteins including B-cell lymphoma-2 (Bcl-2) and $\mathrm{Bcl}-2$-associated $\mathrm{X}(\mathrm{Bax})$ in liver tissues of mice. The study demonstrated that TASP treatment protects against ethanol-induced hepatic injury via multiple pathways by inhib-
\end{abstract}

Correspondence to: Professor Dae-Ki Kim, Department of Immunology and Institute of Medical School, Medical School, Chonbuk National University, 567 Baekje-daero, Deokjin-gu, Jeonju, Jeonbuk 54907, Republic of Korea

E-mail: daekim@jbnu.ac.kr

Professor Young-Mi Lee, Department of Oriental Pharmacy, College of Pharmacy and Wonkwang-Oriental Medicine Research Institute, Wonkwang University, Iksan, Jeonbuk 54538, Republic of Korea E-mail: ymlee@wku.ac.kr

Key words: polysaccharide, Triticum aestivum sprouts, hepatic steatosis, antioxidant, apoptosis iting steatosis and improving antioxidant marker levels during hepatic injury. Such properties provide a basis for therapeutic agents against alcohol-induced liver injury.

\section{Introduction}

Alcohol consumption is the most common risk factor of liver damage; and the main cause of liver disease. Alcoholic liver disease (ALD) is an important global health issue as alcohol consumption leads to hepatic steatosis, hepatitis, life-threatening cirrhosis, and progressive fibrosis (1). Nowadays, many compounds such as corticosteroids are used to treat ALD, but these drugs have adverse effects, which include an elevated risk of infection, gastritis and osteoporosis (2). Accordingly, there is need for more effective drugs with fewer side effects to treat ALD.

The liver is the main organ responsible for the metabolism of alcohol, and many metabolites, such as, acetaldehyde; and toxic lipid species, are formed during this process $(3,4)$. Several mechanisms, such as, steatosis, oxidative stress and inflammatory factors, contribute to the pathogenesis of ALD. Hepatic steatosis is the first manifestation of ALD, and is characterized by lipid accumulation in hepatocytes (5). Much evidence supports the role of oxidative stress in the pathogenesis of ALD (6). Cytochrome P450 2E1 (CYP2E1) catalyzes the conversion of ethanol into acetaldehyde and is markedly overexpressed in ALD (7). In the presence of ethanol exposure, CYP2E1 causes the production of reaction oxygen species (ROS) and depletes glutathione (GSH) (8). In recent studies, NADPH oxidase (NOX) has been identified as a major source of ROS production and an important cause of superoxide dismutase (SOD) production. Furthermore, associations between NOX1 and p67phox, p47phox and p22phox have been associated with alcoholic steatohepatitis, lipid accumulation and hepatic apoptosis (9). NF-E2-related factor 2 (Nrf2) has been reported to play a major role in defense against oxidative stress (10). In addition, it has been shown that Nrf2 is regulated by the phosphatidylinositol 3-kinase (PI3K)/Akt pathway (11), and that excessive ROS production causes mitochondrial damage, cytochrome $c$ (cyt $c$ ) secretion, caspase activation and liver apoptosis (12). 
Triticum aestivum (TA) is a major worldwide crop and an excellent source of biologically active substances $(13,14)$. Previous studies on the pharmacological properties of extracts of Triticum aestivum have reported anti-inflammatory, antioxidative, anti-obesity and anticancer effects (15-19). A previous study revealed that TA-derived phenolic compounds possess antioxidant effects as they produce high free-radical-scavenging activity and SOD-like activity (20). In addition, a recent study showed that TA-derived polysaccharides contain $\beta$-glucan which reduces hyperglycemia and controls diabetes $(21,22)$. Many studies have shown that polysaccharides from different biological sources protect against hepatic fibrosis and injury due to their anti-inflammatory and antioxidant effects $(23,24)$. However, no previous study has examined the hepatoprotective effect of Triticum aestivum sprout-derived polysaccharide (TASP) on ethanol-induced liver injury or the mechanisms involved.

This study was performed to evaluate the effect of TASP on ethanol-induced liver damage in C57BL/6 mice, and to elucidate the molecular mechanisms responsible for its effects.

\section{Materials and methods}

Materials and reagents. Ethanol was purchased from Merck Millipore (Darmstadt, Germany). Silymarin and Oil Red O (ORO) staining were purchased from Sigma (St. Louis, MO, USA). TRIzol reagent and the SuperScript III kit were obtained from Invitrogen (Carlsbad, CA, USA). The protein assay kit (RIPA buffer) was from Santa Cruz Biotechnology, Inc. (Santa Cruz, CA, USA). The primary antibodies, rabbit anti-[p-Akt (9271), Akt (9272) and Bcl-2 (2870)] were from Cell Signaling Technology (Danvers, MA, USA). Rabbit anti-[caspase-3 (sc-7148) and Bcl-2-associated X (Bax) (sc-6236)], mouse anti-[ $\beta$-actin (sc-47778) and CYP2E1 (sc-133491)], and the protein assay kit (RIPA buffer) were obtained from Santa Cruz Biotechnology, Inc. Primary antibodies at 1:1,000 and secondary antibodies at 1:5,000 dilutions were used. For rabbit primary antibodies: goat-antirabbit IgG HRP (\#sc-2030) and for mouse primary antibodies: goat-anti-mouse IgG HRP (\#sc-2030) (both from Santa Cruz Biotechnology) were used.

Extraction and purification of TASP. Triticum aestivum sprouts were supplied by the National Institute of Crop Science (Jeonbuk, Korea) and were freeze-dried. The extraction of water soluble polysaccharides from Triticum aestivum sprouts (TASP) has been previously described $(16,17)$. Briefly, wheatgrass was crushed, powdered, frozen and extracted with ethanol. The extract so obtained was purified with a DEAE Sephadex A-25 column (using 0.15 M sodium chloride as the mobile phase) by high performance liquid chromatography (HPLC; Waters, Milford, MA, USA) equipped with an electrochemical detector (ELCD). The purified extract was precipitated with ethanol for desalting, and the content of TASP was measured using D-glucose as a standard using the phenol-sulfuric acid method $(22,25)$. The extract contained $98 \pm 1 \%$ total carbohydrate and $93 \pm 1 \%$ non-starch polysaccharide. It contained glucose, mannose, and galactose at a ratio of 1.4:1.0:2.1 as determined by neutral sugar composition analysis (22).
Animals and treatment. Male C57BL/6 mice (6-8 weeks old) were purchased from Samtako Bio Korea (Osan, Korea). The experimental procedures were conducted using a protocol approved by the Institutional Animal Care Committee of Chonbuk National University. Mice were housed at $22 \pm 2^{\circ} \mathrm{C}$, $50 \pm 5 \%$ RH and were provided a normal diet. After an acclimatization period of one week, mice were divided into six groups of 6 mice/group. All mice were treated for 10 days by oral gavage: i) normal, mice fed with phosphate-buffered saline (PBS); ii) EtOH, mice fed with 5\% EtOH daily; iii) EtOH+silymarin, mice fed with EtOH and treated with silymarin (100 mg/kg); iv) EtOH+TASP (50), mice fed with EtOH plus TASP $(50 \mathrm{mg} / \mathrm{kg})$ treatment; v) EtOH+TASP (100), mice fed with EtOH plus TASP $(100 \mathrm{mg} / \mathrm{kg})$ treatment; vi) EtOH+TASP (200), mice fed with EtOH plus TASP (200 mg/kg) treatment. On the 11th day, all mice, except mice in the normal control group, were administered an additional single dose of $20 \% \mathrm{EtOH}$, and $12 \mathrm{~h}$ later mice were sacrificed under anesthesia. Blood and liver tissue were collected for further experiments. Recent studies used silymarin as a positive control to investigate the hepatoprotective effect of natural compounds $(26,27)$. Here, we selected silymarin with a dose of $100 \mathrm{mg} / \mathrm{kg}$ as a positive control as it was reported that silymarin, with this dose, significantly reduced the alcoholinduced liver steatosis in mice (26).

Measurement of liver index (\%). Total body weights of mice were measured immediately before sacrifice. Liver weight was measured and the liver index percentage (\%) was calculated by expressing liver weight as the percentage of body weight.

Oil Red $O(O R O)$ staining. Hepatic lipid accumulation was measured by ORO staining. Briefly, portions of left lobes were immediately fixed in $10 \%$ neutral buffered formalin, embedded in frozen section and cut serially into $10-\mu \mathrm{m}$ sections, which were then stained with Oil Red O staining. Pathological changes were observed under a microscope. To measure total lipid, liver tissues were homogenized in a chloroform/methanol mixture $(2: 1, \mathrm{v} / \mathrm{v})$, and total triglycerides (TG) and total cholesterol (TC) were measured using a commercially available kit (Asian Pharmaceutical, Hwaseong-Si, Korea).

Hematoxylin and eosin $(H \& E)$ staining. Liver tissues were fixed with $10 \%$ neutral buffered formalin, embedded in paraffin wax and cut serially into $10-\mu$ m-thick sections, which were then stained with H\&E. Histopathological alterations were observed under a microscope, and photographed (magnification, x100, Olympus CX21; Olympus America Inc., Melville, NY, USA).

Liver enzyme analysis. Blood samples were centrifuged to obtain serum, and serum samples were subjected to biochemical analysis. Serum enzyme activities of alanine aminotransferase (ALT) and aspartate aminotransferase (AST) were measured using ALT/AST cassette test kit (Alere Cholestech LDX $^{\circledR}$ system; Alere Inc., San Diego, CA, USA).

Measurement of hepatic GSH. Immediately after removal, liver tissues were washed in cold isotonic saline. After removing excess saline by blotting, tissues were weighed 
and homogenized in ice-cold 5\% (w/v) meta-phosphoric acid (20 $\mathrm{ml} / \mathrm{g}$ tissue). Homogenates were centrifuged at 14,000 x g for $15 \mathrm{~min}$ at $4^{\circ} \mathrm{C}$ and supernatants were stored at $-80^{\circ} \mathrm{C}$ for future use. Glutathione concentrations in liver tissue were measured using the Glutathione (Total) kit (Enzo Life Sciences, Inc., Farmingdale, NY, USA).

Measurement of hepatic SOD. To measure SOD activities, liver tissues were kept in cold PBS after removal. After repeated washing and blotting, tissues were homogenized and cell pellets were collected. Cytosol extracts were prepared and SOD activities were measured using a SOD assay kit (Dojindo Molecular Technologies, Inc., Rockville, MD, USA) by measuring absorbance at $450 \mathrm{~nm}$ using a microplate reader (Zenyth 200rt; Anthos, Salzburg, Austria).

Real-time PCR. To determine the relative mRNA expression of genes involved in oxidation, we performed real-time PCR using the comparative CT method (28). Total RNA was extracted from liver tissues using TRIzol ${ }^{\circledR}$ RNA isolation reagent, according to the manufacturer's instructions. cDNA was synthesized, using the SuperScript ${ }^{\mathrm{TM}}$ III First Strand Synthesis system (Invitrogen). Real-time PCR was performed using the ABI Real-Time PCR system (Applied Biosystem Inc., Foster City, CA, USA) and SYBR-Green PCR Master Mix (Life Technologies, Carlsbad, CA, USA). The temperature profile used for PCR was $95^{\circ} \mathrm{C}$ for $10 \mathrm{~min}$, followed by 40 cycles of denaturation at $95^{\circ} \mathrm{C}$ for $15 \mathrm{sec}$, and annealing for $60^{\circ} \mathrm{C}$ for $1 \mathrm{~min}$ (29). Glyceraldehyde 3-phosphate dehydrogenase (GAPDH) was the internal control. The primer sequences used were: GAPDH, sense (5'-CAT GGC CTT CCG TGT TC-3') and antisense (5'-CCT GGT CCT CAG TGT AGC-3'); Nrf2, sense (5'-ACC AAG GGG CAC CAT ATA AAA G-3') and antisense (5'-CTT CGC CGA GTT GCA CTC A-3'); hemeoxygenase-1 (HO-1), sense (5'-CAG AAC CAG CCT GAA CTA GC-3') and antisense (5'-TGG ATG TGT ACC TCC TTG GT-3'); CYP2E1, sense (5'-CGT TGC CTT GCT TGT CTG GA-3') and antisense (5'-AAG AAA GGA ATT GGG AAA GGT CC-3'); p67phox, sense (5'-TCT CAT GCA TGC CAA GAA AG-3') and antisense (5'-CTT CAT GTT GGT TGC CAA TG-3'); p47phox, sense (5'-GTC CCT GCA TCC TAT CTG GA-3') and antisense (5'-ATG ACC TCA ATG GCT TCA CC-3') and p22phox, sense (5'-GTG GAC TCC CAT TGA GCC TA-3') and antisense (5'-GTG GAC TCC CAT TGA GCC TA-3').

Western blot analysis. Liver tissues were lysed in ice-cold RIPA buffer for $40 \mathrm{~min}$ and centrifuged $(12,000 \mathrm{x} \mathrm{g})$ for $20 \mathrm{~min}$ at $4^{\circ} \mathrm{C}(30,31)$. Briefly, $30 \mu \mathrm{g}$ of lysates were run on sodium dodecyl sulfate-polyacrylamide gel electrophoresis and transferred to polyvinylidene difluoride (PVDF) membranes (Amersham Pharmacia Biotech, Piscataway, NJ, USA). Membranes were then blocked with 5\% skimmed milk in Tris-buffered saline containing $0.1 \%$ Tween-20 (TBST) for $1 \mathrm{~h}$ at room temperature (RT), probed with primary antibodies at $4^{\circ} \mathrm{C}$ overnight, washed with TBST four times, and incubated with horseradish peroxidase-conjugated secondary antibody for $45 \mathrm{~min}$ at RT. After three washes with TBST, proteins were visualized using an enhanced chemiluminescence detection kit (Millipore, Billerica, MA, USA).
Table I. TASP improves the liver index $(\%)$ in ethanol fed mice.

\begin{tabular}{lccc}
\hline Group & $\begin{array}{c}\text { Body } \\
\text { weight }(\mathrm{g})\end{array}$ & $\begin{array}{c}\text { Liver } \\
\text { weight }(\mathrm{g})\end{array}$ & $\begin{array}{c}\text { Liver } \\
\text { index }(\%)\end{array}$ \\
\hline Control & $23.7 \pm 0.8$ & $1.07 \pm 0.04$ & $4.5 \pm 0.2$ \\
EtOH & $22.4 \pm 0.6$ & $1.20 \pm 0.05$ & $5.3 \pm 0.3$ \\
EtOH+silymarin & $22.0 \pm 0.7$ & $1.07 \pm 0.05$ & $4.9 \pm 0.2^{\mathrm{b}}$ \\
EtOH+TASP (50) & $23.0 \pm 1.2$ & $1.16 \pm 0.04$ & $5.0 \pm 0.3$ \\
EtOH+TASP (100) & $22.9 \pm 0.8$ & $1.12 \pm 0.06$ & $4.9 \pm 0.4^{\mathrm{a}}$ \\
EtOH+TASP (200) & $23.0 \pm 0.6$ & $1.09 \pm 0.05$ & $4.7 \pm 0.4^{\mathrm{b}}$
\end{tabular}

Results are means \pm SEMs $(\mathrm{n}=6) .{ }^{\mathrm{a}} \mathrm{p}<0.05$ and ${ }^{\mathrm{b}} \mathrm{p}<0.01$ vs. the EtOH group. EtOH, ethanol; TASP, Triticum aestivum sprout-derived polysaccharide.

Terminal deoxynucleotidyl transferase dUTP nick end labeling (TUNEL) assay. Hepatic apoptosis was determined using a commercially available TUNEL assay kit (Millipore). Briefly, liver tissues were fixed in $10 \%$ buffered formalin and 5- $\mu \mathrm{m}$ thick frozen sections were obtained, and mounted on slides. Slides were washed in PBS for $30 \mathrm{~min}$ at $37^{\circ} \mathrm{C}$, and incubated with proteinase $\mathrm{K}$ for $30 \mathrm{~min}$ at $37^{\circ} \mathrm{C}$. Slides were rinsed in $\mathrm{PBS}$, treated with TUNEL reaction mixture containing terminal deoxynucleotidyl transferase in a humidified chamber at $37^{\circ} \mathrm{C}$ for $60 \mathrm{~min}$ in the dark, washed with PBS, blocked with avidin-FITC in blocking buffer for $30 \mathrm{~min}$ at $37^{\circ} \mathrm{C}$ in the dark, glass cover slipped, and analyzed under a fluorescent microscope (magnification, x400, Laser Scanning Microscope, LSM 510).

Statistical analysis. Results are expressed as means \pm SEMs, and were analyzed using GraphPad Prism software (version 5.0; GraphPad Software, San Diego, CA, USA). For the evaluation of samples with normal distribution, we used the two-tailed unpaired Student's t-test; otherwise, the non-parametric data were measured by Mann-Whitney test to obtain p-values. p-values $<0.05$ were considered statistically significant.

\section{Results}

TASP improves the liver indices of ethanol fed mice. Body weights, liver weights, and liver indices were measured and compared (Table I). The mean liver index in the ethanol group was $17.8 \%$ higher than that in the normal controls. TASP co-treatment at 50,100 and $200 \mathrm{mg} / \mathrm{kg}$ reduced this increase in mean lipid index to $5.7,7.5$ and $11.3 \%$, respectively.

TASP prevents ethanol-induced hepatic steatosis and improves liver enzymes. To assess the role of TASP in hepatic steatosis induced by ethanol intake, the qualitative measurement of hepatic lipid accumulation was obtained by ORO staining and by measuring TG and TC levels in liver tissues using a commercial kit (Asian Pharmaceutical, Hwaseong-Si, Korea). The ethanol group was found to show neutral lipid droplet accumulation in liver tissues as compared with the normal controls, whereas ORO staining showed that this accumulation 


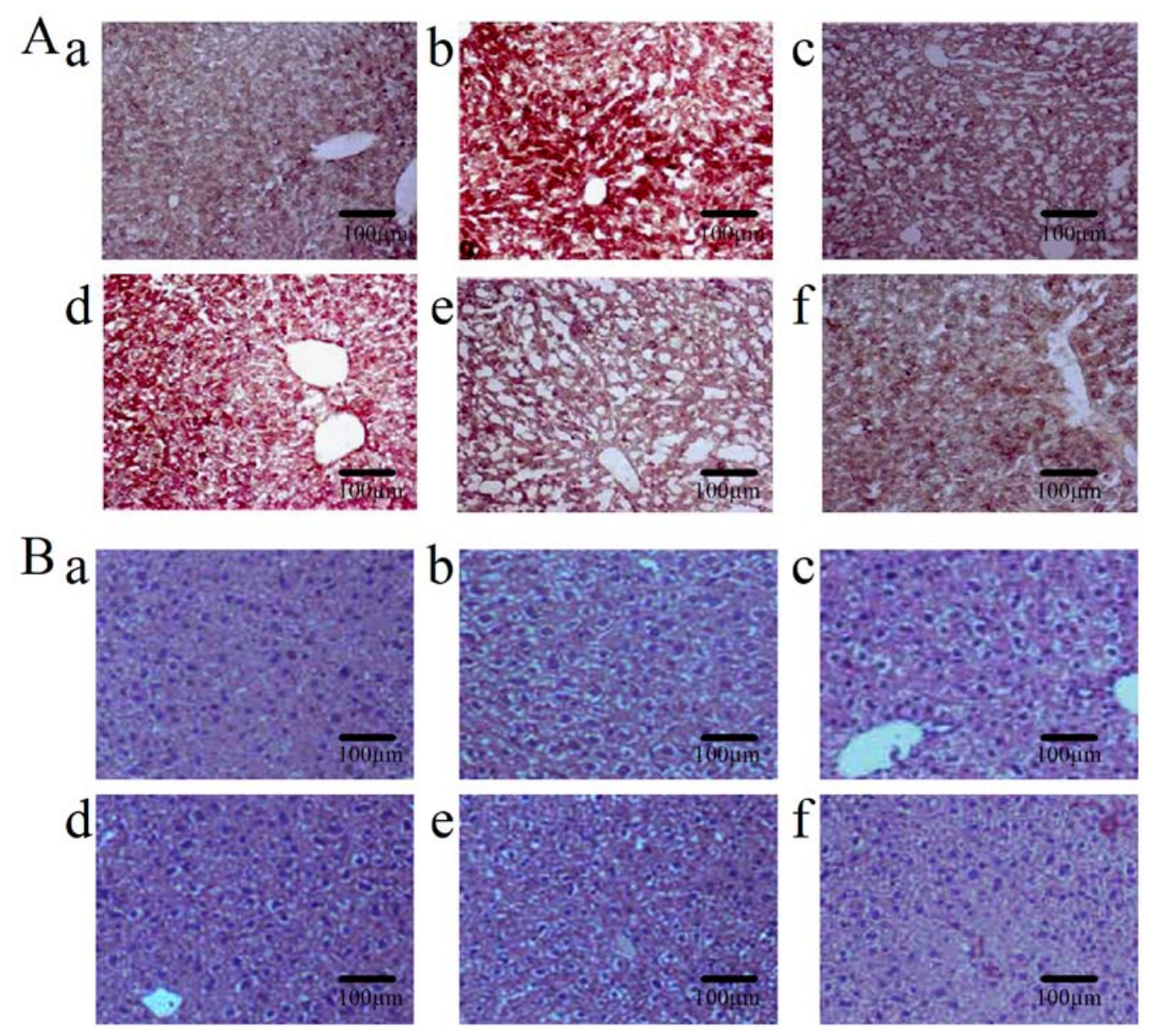

Figure 1. TASP inhibits ethanol-induced hepatic steatosis and improves histological damage. Representative images showing (A) ORO staining, and (B) H\&E staining (original magnification, x100) of (a) treatment naïve control; (b) EtOH control; (c) EtOH+silymarin (100 mg/kg); (d) EtOH+TASP (50 mg/kg); (e) EtOH+TASP (100 mg/kg) and (f) EtOH+TASP (200 mg/kg) groups. EtOH, ethanol; TASP, Triticum aestivum sprout-derived polysaccharide; ORO, Oil Red $\mathrm{O}$ staining; $\mathrm{H} \& \mathrm{E}$, hematoxylin and eosin staining.

was markedly reduced in the three TASP groups (Fig. 1A). Histological analysis of excised livers was performed by using H\&E staining. As shown in Fig. 1B, livers in the control group had a normal architecture, whereas livers in the ethanol group showed excessive vacuolization and ballooning of liver tissues with loss of hepatic architecture, and disappearance of nuclei, but these histological changes were less pronounced in the TASP-treated groups. Quantitative measurements of TG and TC levels in the liver tissues confirmed these histological results and showed a significant increase in lipid accumulation in the ethanol fed group as compared with the normal controls. TASP dose-dependently inhibited the TG and TC increases caused by ethanol treatment and also TASP $(200 \mathrm{mg} / \mathrm{kg})$ more significantly inhibited these increases than that of the silymarin group (Fig. 2A and B). To determine the effect of the TASP co-treatment on ethanol-induced hepatotoxicity, we measured the serum activities of ALT and AST, which are the clinical markers of liver damage. It was found that serum ALT and AST activities were 54.4 and 104\% higher, respectively, in the ethanol group than these values in the normal control (Fig. 2C and D) and that co-treatment with TASP at $200 \mathrm{mg} / \mathrm{kg}$ reduced these increases to 44.3 and $46.8 \%$, respectively, of the ethanol group. These data showed that TASP improved liver steatosis and liver enzymes.
TASP improves the antioxidative status in ethanol-treated mice. The activities of antioxidant enzymes in liver tissues of mice were measured to investigate the protective effect of TASP on ethanol-induced oxidative damage. As shown in Fig. 3, GSH and SOD activities were significantly lower in the ethanol group than these values in the normal controls. However, TASP co-treatment significantly and dose-dependently increased these reductions, and $200 \mathrm{mg} / \mathrm{kg}$ of TASP co-treatment had a greater effect than that noted in the silymarin group.

NOX is the major source of ROS which is involved in hepatic steatatosis and apoptosis $(9,32)$. We determined the effect of TASP on the genes of NOX1, such as p67phox, p47phox and p22phox. The results showed that ethanol fed mice showed increased expression levels of p67phox, p47phox and p22phox than these levels in the normal mice, whereas co-treatment of TASP significantly decreased these increments in NOX1 genes compared to the ethanol group (Fig. 4).

We also performed real-time PCR to assess the mRNA expression of oxidative stress markers. TASP was found to significantly and dose-dependently enhance the mRNA expression of Nrf2 and HO-1 (Fig. 5A and B). Ethanol increased both the mRNA and protein expression of CYP2E1 and these increases were dose-dependently reduced by co-treatment of 

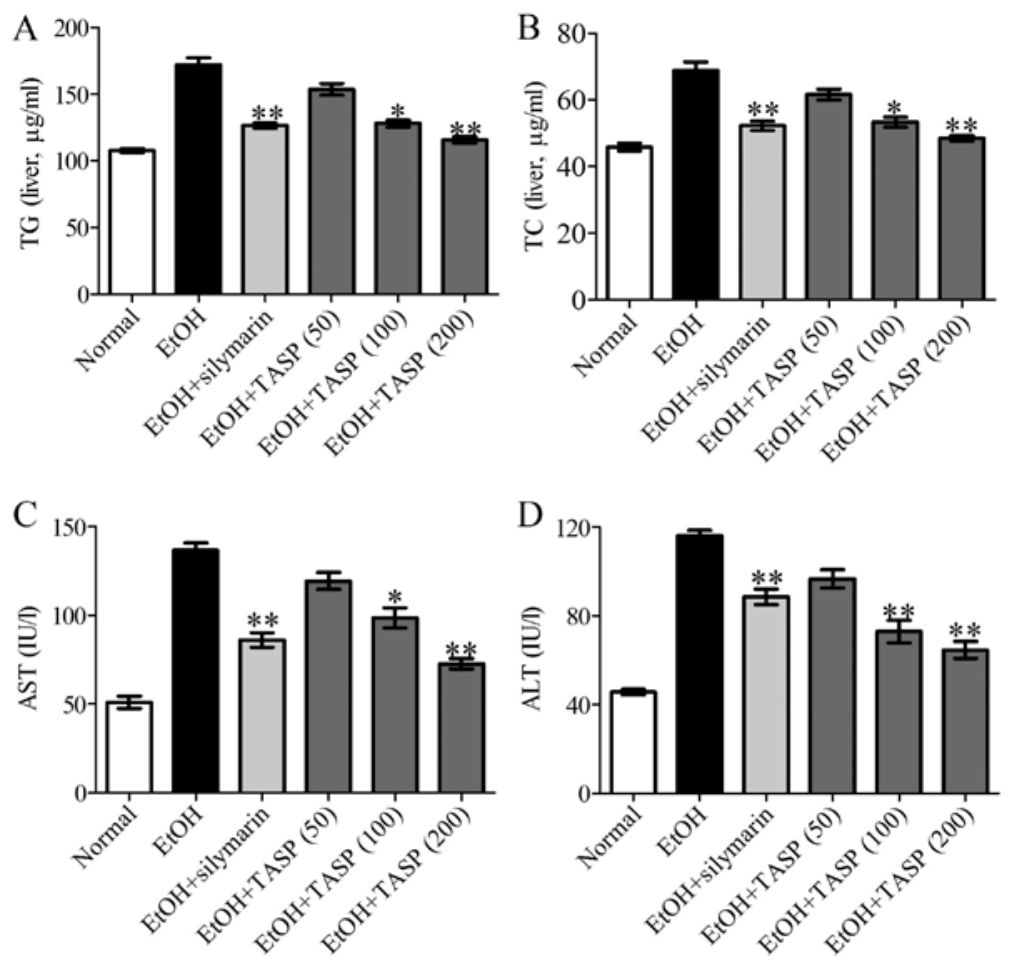

Figure 2. TASP inhibits hepatic lipid accumulation and serum aminotransferase activity in mice administered ethanol. Lipid accumulation was determined quantitatively by measuring hepatic (A) TG and (B) TC levels. Serum levels of (C) AST and (D) ALT were measured using a commercial kit. Results are expressed as means \pm SEMs $(\mathrm{n}=6)$. ${ }^{*} \mathrm{p}<0.05$ and $^{* *} \mathrm{p}<0.01$ vs. the EtOH group. EtOH, ethanol; TASP, Triticum aestivum sprout-derived polysaccharide; AST, aspartate aminotransferase; ALT, alanine aminotransferase; TG, total triglyceride; TC, total cholesterol.
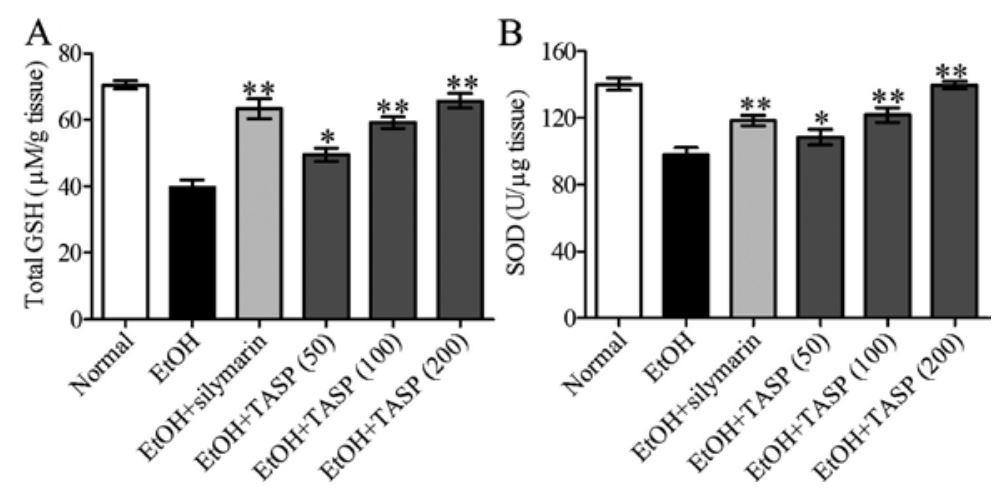

Figure 3. TASP enhances hepatic GSH and SOD activities in mice administered ethanol. Liver tissues were assayed for (A) GSH and (B) SOD activity using a commercial kit. Results are expressed as the means \pm SEMs $(n=6)$. ${ }^{*} p<0.05$ and ${ }^{* *} \mathrm{p}<0.01$ vs. the EtOH group. TASP, Triticum aestivum sprout-derived polysaccharide; LPS, lipopolysaccharide; GSH, total glutathione; SOD, superoxide dismutase.
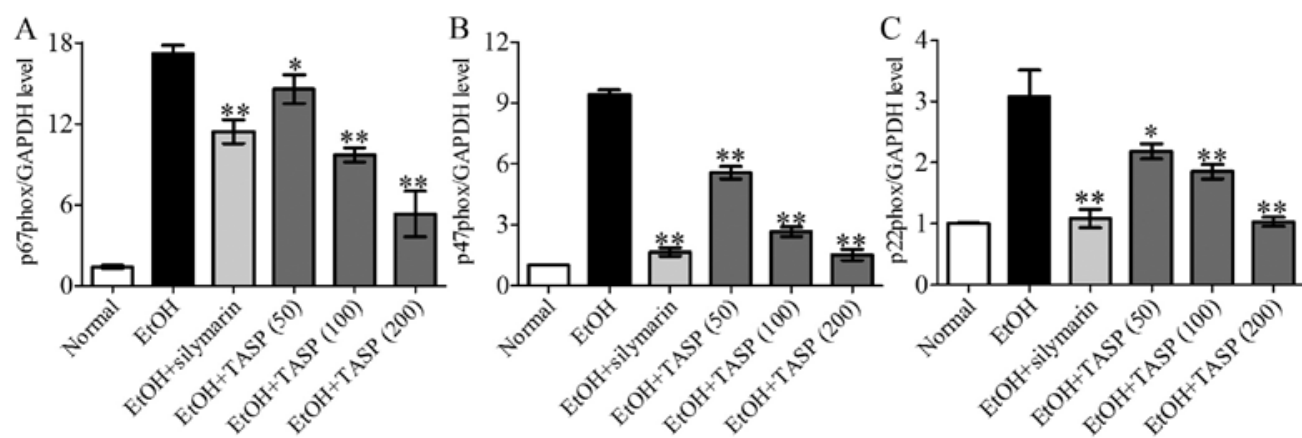

Figure 4. TASP inhibits mRNA expression of NADPH oxidase in ethanol fed mice. The mRNA expression of (A) p67phox, (B) p47phox and (C) p22phox was determined by real-time PCR. Results are expressed as the means \pm SEMs $(\mathrm{n}=6)$. ${ }^{*} \mathrm{p}<0.05$ and ${ }^{* *} \mathrm{p}<0.01$ vs. the EtOH group. TASP, Triticum aestivum sprout-derived polysaccharide. 

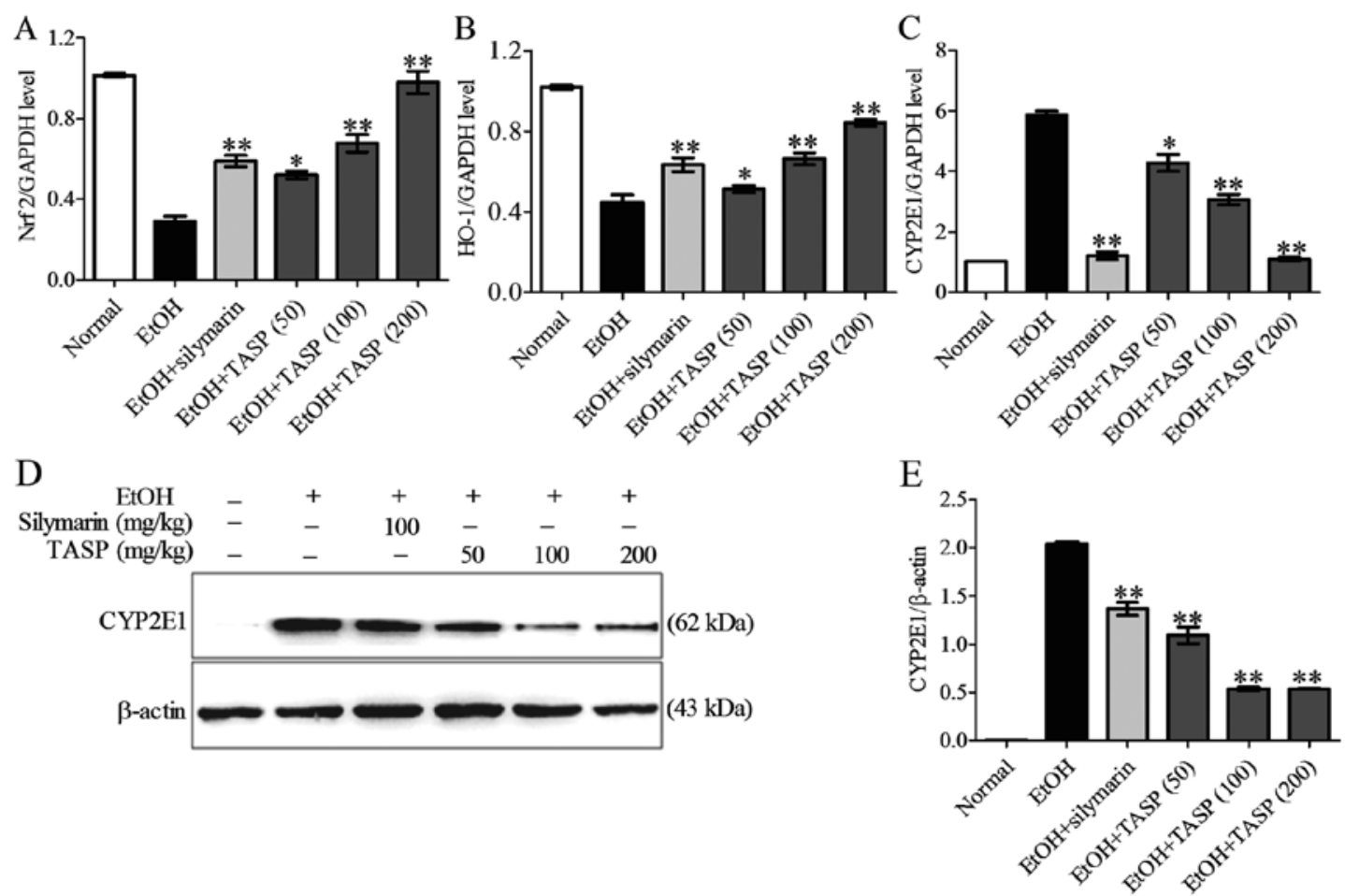

Figure 5. TASP upregulates antioxidant markers in mice administered ethanol. The mRNA expression of (A) Nrf-2, (B) HO-1 and (C) CYP2E1 was determined by real-time PCR. (D) Immunoblot analysis results for CYP2E1 as determined by western blotting and (E) a bar diagram showing the relative band intensities of the immunoblotting. Results are expressed as the means \pm SEMs $(\mathrm{n}=6) .{ }^{*} \mathrm{p}<0.05$ and ${ }^{* *} \mathrm{p}<0.01 \mathrm{vs}$. the EtOH group. TASP, Triticum aestivum sprout-derived polysaccharide; Nrf2, nuclear factor erythroid 2- related factor 2; HO-1, heme oxygenase-1; CYP2E1, cytochrome P450 2E1.
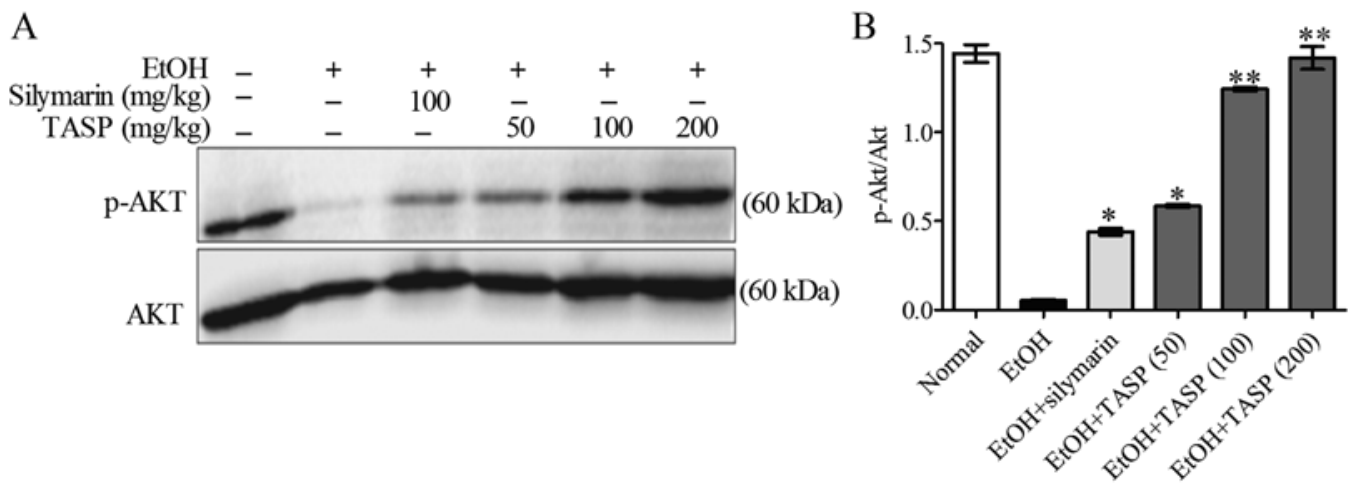

Figure 6. TASP upregulates PI3K/AKT signaling in the liver tissues of mice administered ethanol. We determined the phosphorylation of Akt using western blotting and the total Akt was used as the control. (A) Immunoblot analysis was conducted to assess the phosphorylation of Akt. (B) Bar diagram showing the relative band intensities of blots obtained from three experiments. Each lane represents a single mouse. Results are expressed as the means \pm SEMs $(n=6)$. " $\mathrm{p}<0.05$ and $^{* *} \mathrm{p}<0.01$ vs. the EtOH group. TASP, Triticum aestivum sprout-derived polysaccharide; Akt, protein kinase B.

TASP (Fig. 5C-E). TASP also dose-dependently reduced the ethanol-induced mRNA expression of NADPH oxidase more significantly than silymarin. In a previous study, the PI3K/Akt pathway was shown to be upstream of Nrf2 and to play an important role in the synthesis of GSH (33). Therefore, to elucidate the mechanism responsible for the antioxidative effect of TASP, we investigated the phosphorylation of Akt. Western blot analysis was performed to determine the phosphorylation of Akt by using total Akt protein expression as a control (32). It was found that ethanol decreased Akt phosphorylation compared to that noted in the normal controls, and TASP significantly and dose-dependently reduced this increase and did so to a significantly greater extent than silymarin (Fig. 6).
TASP reduces ethanol-induced apoptosis in liver tissues. TUNEL staining was used to examine apoptotic bodies in the liver tissues. A large number of TUNEL-positive hepatocytes were observed in the ethanol group, but these numbers were significantly and dose-dependently reduced by TASP (Fig. 7).

We also examined the effect of TASP on the protein level of caspase-3 (a predominant downstream effector of apoptosis activated by caspases- 8 and -9 , which trigger apoptosis via extrinsic and intrinsic pathways, respectively) (12). As shown in Fig. 8, caspase-3 activation was detected using western blot analysis showing that the top band corresponds to full-length caspase- 3 and the bottom band to cleaved caspase-3. Caspase-3 protein levels were significantly higher 

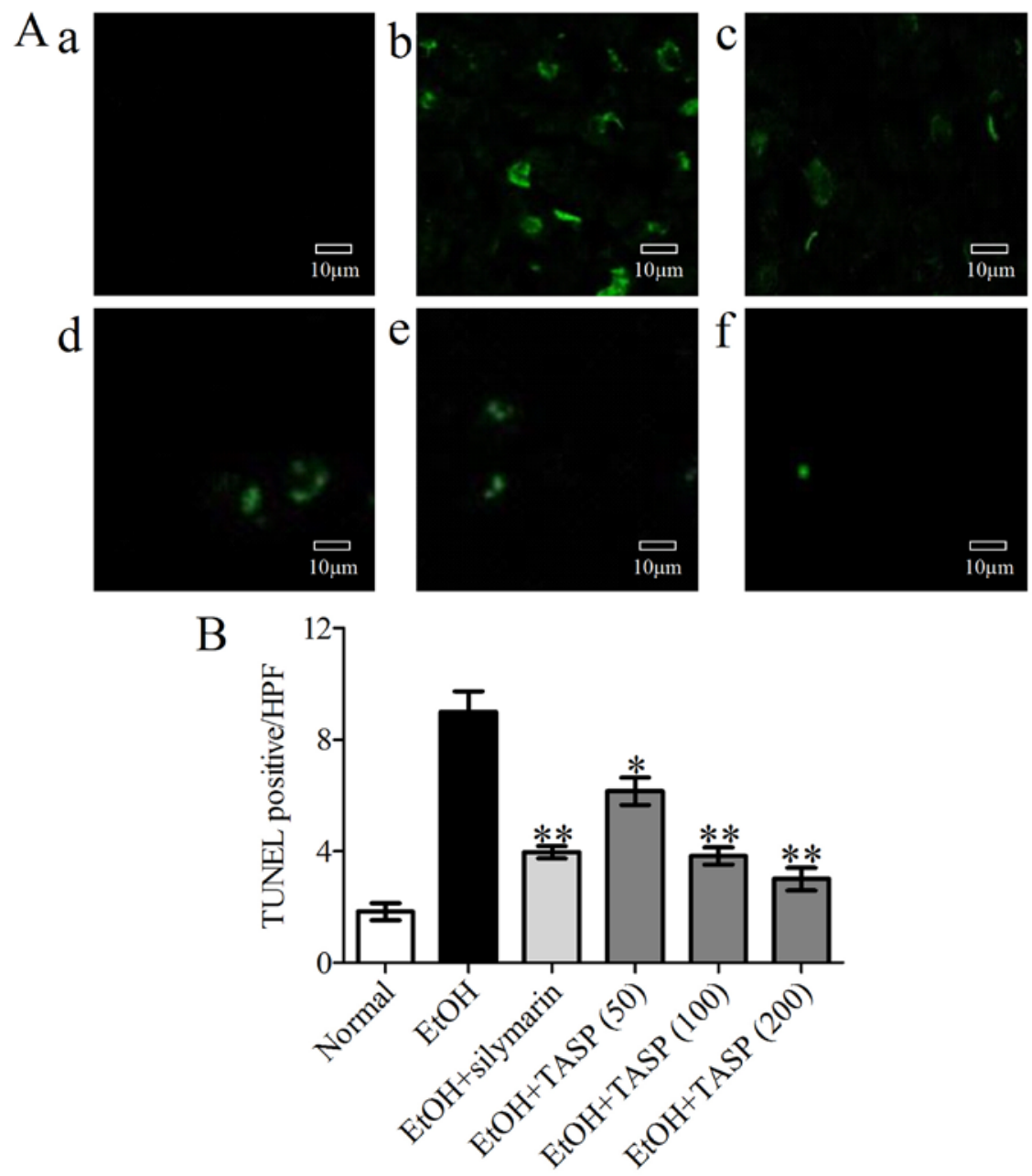

Figure 7. TASP suppresses the number of TUNEL-positive hepatocytes in mice administered ethanol. (A) TUNEL-positive cells in (a) normal control; (b) EtOH control; (c) EtOH+silymarin (100 mg/kg); (d) EtOH+TASP (50 mg/kg); (e) EtOH+TASP (100 mg/kg) and (f) EtOH + TASP (200 mg/kg) groups. Magnification, $\mathrm{x}$ 400. (B) Apoptosis index was defined as the number of apoptotic cells present in a high power field. Results are expressed as the means \pm SEMs $(\mathrm{n}=6) .{ }^{*} \mathrm{p}<0.05$ and $^{* *} \mathrm{p}<0.01$ vs. the EtOH group. TASP, Triticum aestivum sprout-derived polysaccharide.

in the ethanol-fed group than that noted in the normal control or ethanol non-fed group, but TASP co-treatment significantly downregulated caspase-3 levels as compared with the ethanol group (Fig. 8A and B). We also found that ethanol significantly increased Bax protein levels in the liver, and that TASP co-treatment significantly suppressed this increase (Fig. 8A and C). In contrast, Bcl-2 protein levels were reduced by ethanol administration, and TASP dosedependently prevented this reduction (Fig. 8A and C). Our results showed that TASP caused a significant decrease in caspase- 3 activation and Bax protein level. In addition, TASP significantly enhanced the Bcl-2 protein level in the liver tissues of the ethanol-fed mice when compared with that of silymarin.

\section{Discussion}

It has been well-established that acute or chronic alcohol consumption causes hepatic injury. Although medications are available to treat ethanol-induced hepatic damage, the associated side effects, for example, in the case of steroids, can cause gastrointestinal symptoms. In contrast, drugs isolated from plants and herbs have few side effects. In a recent study, TASP was found to reduce hyperlipidemia and improve insulin resistance $(21,22)$.

A study showed that polysaccharides derived from plants protect against hepatic fibrosis and inhibit inflammation (23). A similar hepatoprotective effect was observed in rats treated with $\beta$-glucan-enriched Euglena gracilis Z. (34). In addition, a recent study showed that a fermentable marine $\beta$-glucan protected against hepatotoxicity induced by LPS through modulation of the immune response (35). However, the hepatoprotective effect of TASP has not been previously studied, and this is the first study to report the hepatoprotective effects of TASP on ethanol-induced liver injury.

In the present study, we did not use polysaccharides from rice or other plants as a negative control but selected the nontoxic concentration in a dose-dependent manner as illustrated by previous studies for determining the hepatoprotective effect of polysaccharide from plants $(23,24)$. Ethanol non-fed mice or normal mice served as the negative control in this study. The absence of polysaccharides from rice or other plants used as a negative control is a limitation of our study. Ethanol-induced hepatic injury was compared with the ethanol non-fed mice and the effect of TASP was compared with ethanol-fed mice. Our result showed that TASP significantly reduced ethanol-induced 


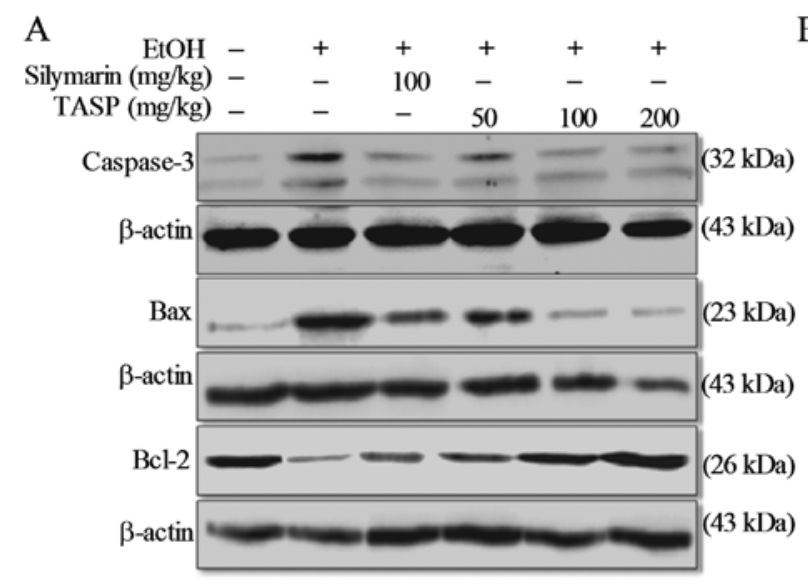

B
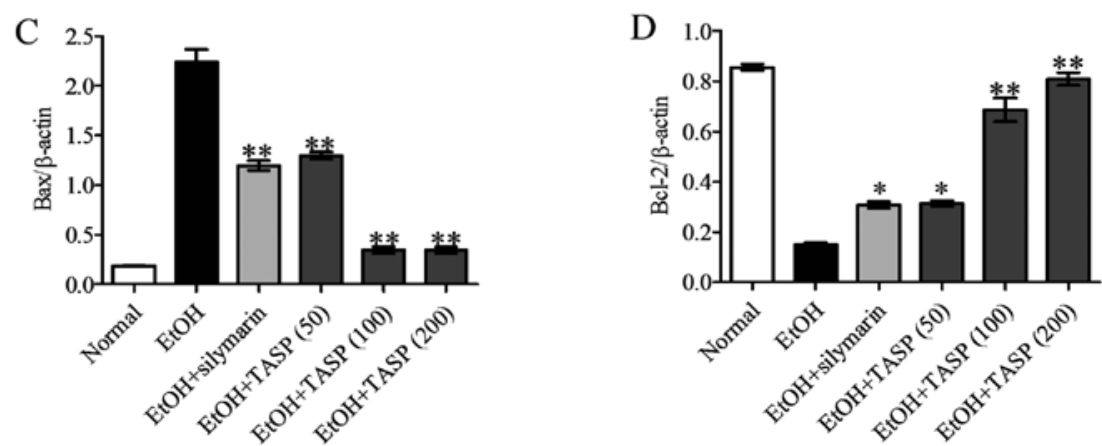

Figure 8. TASP inhibits apoptosis in mice administered ethanol. (A) Immunoblot analysis of apoptosis markers. (B-D) Bar diagrams showing the relative band intensities of blots from three experiments. Each lane represents a single mouse. Results are means \pm SEMs $(n=6)$. ${ }^{*}<<0.05$ and ${ }^{* *} p<0.01 \mathrm{vs.} \mathrm{the} \mathrm{EtOH} \mathrm{group.}$ TASP, Triticum aestivum sprout-derived polysaccharide; Bcl-2, apoptosis associated mitochondrial proteins, B-cell lymphoma-2; Bax, Bcl-2-associated X.

hepatic steatosis as evidenced by reduced ORO staining and total lipid and total cholesterol levels in liver tissues. These findings are similar to those reported for the effect of luteolin (a phytoflavone) on ethanol-induced liver injury (5).

Elevated serum activities of aminotransferase ALT and AST are prominent signs of hepatic damage. In the present study, TASP reduced ethanol-induced ALT and AST levels more significantly than silymarin, a drug used to treat liver injury. Moreover, H\&E staining showed that TASP improved hepatic architecture by preventing excessive vacuolization, loss of hepatic architecture, and disappearance of nuclei. In line with our results, it was previously reported that inhibition of ALT and AST improved architecture in a mouse model of alcohol-induced liver steatosis and injury (26).

GSH and SOD are antioxidant enzymes and scavengers of free radicals and ROS in liver, and are the first line of defense against oxidative injury. GSH plays an important role in protection from oxidative stress by removing lipids and organic peroxides (36), whereas SOD defends against oxidative stress by removing superoxide radicals (37). Alcohol increases oxidative stress by increasing the intracellular levels of reactive oxygen species and by reducing the activities of GSH and SOD $(37,38)$. Our results showed that oral co-treatment with TASP enhanced GSH and SOD activities in liver tissues more significantly than silymarin in the ethanol fed mice. Similarly, it was previously reported that GSH and SOD augmentation reduced acute alcohol-induced oxidative damage in mice (39). In addition, we observed that TASP reduced ethanol-induced NOX (a primary source of SOD production) more significantly than silymarin.
CYP2E1 is a member of the cytochrome P450 enzyme family, and when active produces ROS. Furthermore, CYP2E is highly expressed by the acute and chronic intake of alcohol $(38,39)$, and plays an important role in alcohol-induced liver injury, by increasing oxidative stress and lipid accumulation in the liver $(40,41)$. On the other hand, Nrf2 is an important transcription factor which induces cytoprotective signals in the presence of oxidative stimuli and also regulates metabolism of hepatic lipid $(42,43)$. In line with other studies, our results showed that TASP significantly decreased ethanol-induced CYP2E1 expression and enhanced the mRNA expression of the antioxidant genes, Nrf2 and HO-1.

Akt is the primary mediator of the PI3K-initiated signaling pathway, and when phosphorylated acts as an antiapoptotic signaling molecule $(33,44)$. Several studies have reported that PI3K/Akt signaling is important for Nrf2 translocation, and thus, for the regulation of GSH (11). According to a previous report, the ratio of p-Akt/Akt showed that the hepatic p-Akt level reduced in alcohol-fed mice was due to the change in the phosphorylation level of p-Akt rather than a change in total protein of Akt (32). Hence we determined the p-Akt/Akt ratio rather than $\mathrm{p}-\mathrm{Akt} / \beta$-actin in this study. TASP showed a hepatoprotective effect in ethanol-induced liver injury by upregulation of Akt phosphorylation which controls antioxidative markers.

Apoptosis is a feature of alcohol-induced liver injury $(8,45)$. Excessive ROS production causes damage to mitochondria and causes leakage of cyt $c$, caspase activation, and apoptosis (12). Many studies have assessed the cell death in liver tissues by TUNEL assay counterstained with 4',6-diamidino-2-phenyl- 
indole (DAPI), a nuclear staining $(46,47)$. In this study, cell death was assessed by TUNEL assay that detects DNA breaks but counterstaining with DAPI was not performed. In the late stage of apoptosis, all nuclei break and DAPI may not be able to stain them anymore (48). Thus, the use of the TUNEL assay is a reliable marker for apoptosis (33).Apoptosis may be more clearly assessed using DAPI as a counterstain in the TUNEL assay but we assessed proteins using only TUNEL assay as it can detect apoptosis easily. Our results showed a large number of TUNEL-positive hepatocytes in the ethanol-treated group. Furthermore, TASP (200 mg/kg) was found to be more effective in this respect than silymarin. Also, we performed western blot analysis to measure key proteins, such as Bax and $\mathrm{Bcl}-2$ associated with apoptosis (33), to further illustrate the effect of TASP in ethanol-induced apoptosis.

Caspase- 3 is the downstream executioner of programmed cell death activated by both the intrinsic and extrinsic pathways $(18,33)$. In the present study, caspase-3 protein level in liver tissues was enhanced by ethanol administration, but this increase was diminished by TASP co-treatment. In addition, TASP decreased caspase- 3 and Bax protein (a pro-apoptotic protein) levels and increased Bcl-2 (an antiapoptotic protein) levels. Similarly, in a previous study, peptides were observed to inhibit hepatocyte apoptosis in an ethanol-induced liver injury model (33).

Taken together, our results suggest that TASP is a natural hepatoprotective agent that protects against alcohol-induced liver damage. The underlying mode of action of TASP appears to involve antioxidant and antiapoptotic effects and reduced hepatic steatosis. Further studies are required to elucidate the molecular mechanisms underlying the protective effects of TASP.

\section{Acknowledgements}

The study was supported by a 2017 grant from Wonkwang University (Jeonbuk, Korea).

\section{References}

1. Park SH, Kim CH, Kim DJ, Park JH, Kim TO, Yang SY Moon YS, Kim TN, Kim HK, Park HY, et al: Prevalence of alcoholic liver disease among Korean adults: Results from the fourth Korea National Health and Nutrition Examination Survey, 2009. Subst Use Misuse 46: 1755-1762, 2011.

2. Arteel G, Marsano L, Mendez C, Bentley F and McClain CJ: Advances in alcoholic liver disease. Best Pract Res Clin Gastroenterol 17: 625-647, 2003.

3. Kawaratani H, Tsujimoto T, Douhara A, Takaya H, Moriya $\mathrm{K}$, Namisaki T, Noguchi R, Yoshiji H, Fujimoto M and Fukui H: The effect of inflammatory cytokines in alcoholic liver disease. Mediators Inflamm 2013: 495156, 2013

4. Zamora Nava LE, Aguirre Valadez J, Chávez-Tapia NC and Torre A: Acute-on-chronic liver failure: A review. Ther Clin Risk Manag 10: 295-303, 2014.

5. Liu G, Zhang Y, Liu C, Xu D, Zhang R, Cheng Y, Pan Y, Huang C and Chen Y: Luteolin alleviates alcoholic liver disease induced by chronic and binge ethanol feeding in mice. J Nutr 144: 1009-1015, 2014.

6. Knockaert L, Descatoire V, Vadrot N, Fromenty B and Robin MA: Mitochondrial CYP2E1 is sufficient to mediate oxidative stress and cytotoxicity induced by ethanol and acetaminophen. Toxicol In Vitro 25: 475-484, 2011.

7. Yamashita H, Goto M, Matsui-Yuasa I and Kojima-Yuasa A: Ecklonia cava polyphenol has a protective effect against ethanolinduced liver injury in a cyclic AMP-dependent manner. Mar Drugs 13: 3877-3891, 2015 .
8. Xu T, Zheng L, Xu L, Yin L, Qi Y, Xu Y, Han X and Peng J: Protective effects of dioscin against alcohol-induced liver injury. Arch Toxicol 88: 739-753, 2014.

9. Jiang JX and Török NJ: NADPH oxidases in chronic liver diseases. Adv Hepatol 2014: 742931, 2014.

10. Xu W, Hellerbrand C, Köhler UA, Bugnon P, Kan YW, Werner S and Beyer TA: The Nrf2 transcription factor protects from toxininduced liver injury and fibrosis. Lab Invest 88: 1068-1078, 2008.

11. Ma X, Zhao YL, Zhu Y, Chen Z, Wang JB, Li RY, Chen C, Wei SZ, Li JY, Liu B, et al: Paeonia lactiflora Pall. protects against ANIT-induced cholestasis by activating Nrf2 via PI3K/Akt signaling pathway. Drug Des Devel Ther 9: 5061-5074, 2015.

12. Neuman MG: Apoptosis in liver disease. Rom J Gastroenterol 11: 3-7, 2002.

13. Lee SH, Xin MJ, Luyen BTT, Cha JY, Im JY, Kwon SU, Lim SW, Suh JW, Kim YH, Kim DK, et al: Inhibitory effect of Triticum aestivum ethanol extract on lipid accumulation in 3T3-L1 preadipocytes. Yakhak Hoeji 55: 478-484, 2011

14. Wojakowska A, Perkowski J, Góral T and Stobiecki M: Structural characterization of flavonoid glycosides from leaves of wheat (Triticum aestivum L.) using LC/MS/MS profiling of the target compounds. J Mass Spectrom 48: 329-339, 2013.

15. Benedetti S, Primiterra M, Tagliamonte MC, Carnevali A, Gianotti A, Bordoni A and Canestrari F: Counteraction of oxidative damage in the rat liver by an ancient grain (Kamut brand khorasan wheat). Nutrition 28: 436-441, 2012.

16. Luyen BT, Tai BH, Thao NP, Cha JY, Lee YM and Kim YH: A new phenolic component from Triticum aestivum sprouts and its effects on LPS-stimulated production of nitric oxide and TNF- $\alpha$ in RAW 264.7 cells. Phytother Res 28: 1064-1070, 2014.

17. Luyen BT, Thao NP, Tai BH, Lim JY, Ki HH, Kim DK, Lee YM and Kim YH: Chemical constituents of Triticum aestivum and their effects on adipogenic differentiation of 3T3-L1 preadipocytes. Arch Pharm Res 38: 1011-1018, 2015.

18. Poudel B, Ki HH, Luyen BT, Lee YM, Kim YH and Kim DK: Triticumoside induces apoptosis via caspase-dependent mitochondrial pathway and inhibits migration through downregulation of MMP2/9 in human lung cancer cells. Acta Biochim Biophys Sin (Shanghai) 48: 153-160, 2016.

19. Poudel B, Nepali S, Xin M, Ki HH, Kim YH, Kim DK and Lee YM: Flavonoids from Triticum aestivum inhibit adipogenesis in 3T3-L1 cells by upregulating the insig pathway. Mol Med Rep 12: 3139-3145, 2015.

20. Yang JY: jeong EY, Kim DK and Lee HS. Antioxidant and $\alpha$-glucosidase inhibitory effects in Triticum aestivum sprouts treated to chilling temperature. J Korean Soc Appl Biol Chem 54: 644-648, 2011.

21. Lee SH, Lim SW, Lee YM, Lee HS and Kim DK: Polysaccharide isolated from Triticum aestivum stimulates insulin release from pancreatic cells via the ATP-sensitive $\mathrm{K}^{+}$channel. Int J Mol Med 29: 913-919, 2012.

22. Lee SH: LY, Lee HS and Kim DK: Anti-oxidative and anti-hyperglycemia effects of Triticum aestivum wheat sprout water extracts on the streptozotocin-induced diabetic mice. Korean J Pharmacogn 40: 408-414, 2009.

23. Zhang K, Gao Y, Zhong M, Xu Y, Li J, Chen Y, Duan X and Zhu H: Hepatoprotective effects of Dicliptera chinensis polysaccharides on dimethylnitrosamine-induced hepatic fibrosis rats and its underlying mechanism. J Ethnopharmacol 179: 38-44, 2016.

24. Liu Q, Zhu M, Geng X, Wang H and Ng TB: Characterization of polysaccharides with antioxidant and hepatoprotective activities from the edible mushroom Oudemanisella radicata. Molecules 22: E234, 2017.

25. Lai DM,Høj PB and Fincher GB: Purification and characterization of $(1 \rightarrow 3,1 \rightarrow 4)$-beta-glucan endohydrolases from germinated wheat (Triticum aestivum). Plant Mol Biol 22: 847-859, 1993.

26. Lian LH, Wu YL, Song SZ, Wan Y, Xie WX, Li X, Bai T, Ouyang BQ and Nan JX: Gentiana manshurica Kitagawa reverses acute alcohol-induced liver steatosis through blocking sterol regulatory element-binding protein-1 maturation. J Agric Food Chem 58: 13013-13019, 2010.

27. Choi MK, Han JM, Kim HG, Lee JS, Lee JS, Wang JH, Son SW, Park HJ and Son CG: Aqueous extract of Artemisia capillaris exerts hepatoprotective action in alcohol-pyrazole-fed rat model. J Ethnopharmacol 147: 662-670, 2013.

28. Schmittgen TD and Livak KJ: Analyzing real-time PCR data by the comparative C(T) method. Nat Protoc 3: 1101-1108, 2008. 
29. Nepali S, Son JS,Poudel B,Lee JH,Lee YM and Kim DK: Luteolin is a bioflavonoid that attenuates adipocyte-derived inflammatory responses via suppression of nuclear factor- $\kappa \mathrm{B} /$ mitogen-activated protein kinases pathway. Pharmacogn Mag 11: 627-635, 2015.

30. Poudel B, Ki HH, Lee YM and Kim DK: Collagen I-induced dendritic cells activation is regulated by TNF-alpha production through down-regulation of IRF4. J Biosci 40: 71-78, 2015.

31. Poudel B, Lim SW, Ki HH, Nepali S, Lee YM and Kim DK: Dioscin inhibits adipogenesis through the AMPK/MAPK pathway in 3T3-L1 cells and modulates fat accumulation in obese mice. Int J Mol Med 34: 1401-1408, 2014.

32. Zhang ZH, Liu XQ, Zhang C, He W, Wang H, Chen YH, Liu XJ, Chen $\mathrm{X}$ and $\mathrm{Xu} \mathrm{DX}$ : Tlr4-mutant mice are resistant to acute alcohol-induced sterol-regulatory element binding protein activation and hepatic lipid accumulation. Sci Rep 6: 33513, 2016.

33. Ma Z, Hou T, Shi W, Liu W and He H: Inhibition of hepatocyte apoptosis: An important mechanism of corn peptides attenuating liverinjury induced by ethanol. Int J Mol Sci 16: 22062-22080, 2015.

34. Sugiyama A, Suzuki K, Mitra S, Arashida R, Yoshida E, Nakano R, Yabuta Y and Takeuchi T: Hepatoprotective effects of paramylon, a beta-1, 3-D-glucan isolated from Euglena gracilis $\mathrm{Z}$, on acute liver injury induced by carbon tetrachloride in rats. J Vet Med Sci 71: 885-890, 2009.

35. Neyrinck AM, Mouson A and Delzenne NM: Dietary supplementation with laminarin, a fermentable marine beta (1-3) glucan, protects against hepatotoxicity induced by LPS in rat by modulating immune response in the hepatic tissue. Int Immunopharmacol 7: 1497-1506, 2007.

36. Fernández-Checa JC, Kaplowitz N, García-Ruiz C, Colell A, Miranda M, Marí M, Ardite E and Morales A: GSH transport in mitochondria: Defense against TNF-induced oxidative stress and alcohol-induced defect. Am J Physiol 273: G7-G17, 1997.

37. Xiong ZE, Dong WG, Wang BY, Tong QY and Li ZY: Curcumin attenuates chronic ethanol-induced liver injury by inhibition of oxidative stress via mitogen-activated protein kinase/nuclear factor E2-related factor 2 pathway in mice. Pharmacogn Mag 11: 707-715, 2015

38. Han Y, Xu Q, Hu JN, Han XY, Li W and Zhao LC: Maltol, a food flavoring agent, attenuates acute alcohol-induced oxidative damage in mice. Nutrients 7: 682-696, 2015.
39. Leung TM and Nieto N: CYP2E1 and oxidant stress in alcoholic and non-alcoholic fatty liver disease. J Hepatol 58: 395-398, 2013.

40. Jimenez-Lopez JM and Cederbaum AI: CYP2E1-dependent oxidative stress and toxicity: Role in ethanol-induced liver injury. Expert Opin Drug Metab Toxicol 1: 671-685, 2005.

41. Lu Y, Wu D, Wang X, Ward SC and Cederbaum AI: Chronic alcohol-induced liver injury and oxidant stress are decreased in cytochrome P4502E1 knockout mice and restored in humanized cytochrome P4502E1 knock-in mice. Free Radic Biol Med 49: 1406-1416, 2010.

42. Huang J, Tabbi-Anneni I, Gunda V and Wang L: Transcription factor Nrf2 regulates SHP and lipogenic gene expression in hepatic lipid metabolism. Am J Physiol Gastrointest Liver Physiol 299: G1211-G1221, 2010.

43. Liu J, Wang X, Liu R, Liu Y, Zhang T, Fu H and Hai C: Oleanolic acid co-administration alleviates ethanol-induced hepatic injury via Nrf-2 and ethanol-metabolizing modulating in rats. Chem Biol Interact 221: 88-98, 2014.

44. Abdelmegeed MA, Banerjee A, Jang S, Yoo SH, Yun JW, Gonzalez FJ, Keshavarzian A and Song BJ: CYP2E1 potentiates binge alcohol-induced gut leakiness, steatohepatitis, and apoptosis. Free Radic Biol Med 65: 1238-1245, 2013.

45. Cao YW, Jiang Y, Zhang DY, Wang M, Chen WS, Su H, Wang YT and Wan JB: Protective effects of Penthorum chinense Pursh against chronic ethanol-induced liver injury in mice. J Ethnopharmacol 161: 92-98, 2015.

46. Gao X, Fan L, Li H, Li J, Liu X, Sun R and Yu Z: Hepatic injury is associated with cell cycle arrest and apoptosis with alteration of cyclin A and D1 in ammonium chloride-induced hyperammonemic rats. Exp Ther Med 11: 427-434, 2016.

47. Ducan MB, Yang C, Tanjore H, Boyle PM, Keskin D, Sugimoto H, Zeisberg M, Olsen BR and Kalluri R: Type XVII collagen is essential for survival during acute injury in mice. Dis Model Mech 4: 942-951, 2013.

48. Collins JA, Schandi CA, Young KK, Vesely J and Willingham MC: Major DNA fragmentation is a late event in apoptosis. J Histochem Cytochem 45: 923-934, 1997. 